\title{
Beyond Pain, Fear, Withdrawal-Findings, And Problems Involving Change - Treatment and Application for $A$ Chronic Addiction on Alcohol Do Not End
}

\author{
Loai Aljerf ${ }^{1 *}$ and Nuha AlMasri ${ }^{2}$ \\ ${ }^{1}$ Department of Basic Sciences, Faculty of Dental Medicine, Damascus University, Damascus, Syria \\ ${ }^{2}$ Department of Chemistry, Faculty of Medicine, Syrian Private University, Damascus, Syria
}

Received: 畊 July 25, 2018; Published: 阱 July 30, 2018

*Corresponding author: Loai Aljerf, Department of Basic Sciences, Faculty of Dental Medicine, Damascus University, Damascus, Syria

\begin{abstract}
Alcohol use disorders (AUD) involving hazardous, harmful and addictive misuse of alcohol are widespread in most parts of the world. The aim of this study was to review the effect of the common inhibitors in the treatment of patients with AUD, taking into consideration, the short- and long- terms abstinence. An extensive literature search conducted in MEDLINE, PubMed, Scopus and CINAHL databases identified 776 articles, which were then evaluated for pre-specified criteria for relevance and quality assurance. A total of 38 articles, including 36 human studies and 2 animal studies, were selected for this review. Many inhibitors used in the treatment of alcoholism and some were considered of effective medication when their intakes are supervised by an expert. However, their therapeutic efficacies vary widely; for instance, disulfiram is a pro-drug that requires its transformation into an active form and because it shows a wide range of secondary effects, it often prevents the use of doses that ensure full therapeutic effectiveness. Sex hormones play an important role in establishing sex-distinctive brain structural and functional variations that could contribute to the sex differences in alcohol consumption behavior. Existing evidence supports the association of increased testosterone level and increased risk for alcohol use and AUD in males. In contrast, the evidence supports the association of increased estrogen level and increased alcohol use in females. Much less is known about the impact of progestins on alcohol use and misuse in human subjects. Future observational and experimental studies conducted in both sexes with a comprehensive hormone panel are needed to elucidate the impact of the interplay between various sex hormone levels during various developmental stages on alcohol userelated phenotypes and AUD. On the other hand, alcohol withdrawal-especially delirium tremens (DT)-is a potentially life-threatening condition. While short-term treatment regimens and factors that predispose to more severe symptomatology have been extensively studied, little attention has been paid to the clinical epidemiology and long-term care of the chronic medical, addictive, psychiatric, and psychosocial problems faced by these patients. Chronic alcoholic patients are frequently suffering from specific micronutrient deficiencies, including vitamins involved in one carbon metabolism. The deficiencies commonly involve folate, vitamin B6, thiamine, and vitamin A. Inadequate dietary intake is a major cause of the vitamin deficiency. As a consequence of chronic alcohol intake, could lead to metabolic disruption and potentially to hyperhomocysteinemia. Alcoholism can affect the absorption, storage, metabolism, and activation of many of these vitamins.
\end{abstract}

Keywords: Chronic Addiction; Alcohol Use Disorders; Alcoholism; Micronutrient deficiencies; Psychiatric Treatment; Physiological Disturbances; Therapeutic Armamentarium

\section{Introduction}

Drugs, of one sort or another, have long been used in the treatment of chronic alcoholism. All sorts of claims had been advanced for the efficacy of various drug therapies in the past, but the short life of their popularity and the invariable realization of

the fact that such drugs made no real difference in the course of the disease were ample proof of the fallacy of these claims. In recent years new drugs have appeared [1], which offer promise in the treatment of chronic alcoholism, but in order to avoid the mistake 
of the past and indeed discourage the use of potentially effective agents in this condition, it would be well to consider the nature of alcoholism and to define just what one should expect from a drug used in its treatment. Chronic alcoholism [2], as we understand it today, is a complex illness involving the whole individual. Proper treatment, in our view, requires the recognition of the emotional and social factors operating in chronic alcoholics as well as the diagnosis of physical and physiological abnormalities resulting from the excessive use of alcohol. Any therapeutic program designed to abolish the abnormal drinking pattern, which is the crux of the problem, must include a positive approach towards the disturbed personality of the alcoholic. Basic in the treatment of the alcoholic is the recognition of the fact that a personality disturbance is always present and that specific measures must be taken and carried out for long periods of time in order to overcome this disturbance. Such measures will vary with the social and emotional backgrounds of the patient and the physician. The only criterion that can be applied to their success is the ability of the patient to maintain sobriety, and the improvement of his social, business and personal relations which result there from.

Approaches such as Alcoholics Anonymous, religious conversions, the counseling of a wise and sympathetic physician Table 1: Drugs used in the treatment of alcoholism. and formal psychiatric treatment are all valuable in accomplishing permanent sobriety and the necessary changes in the personality [2]. The choice will be dependent on the feeling of the individual patient and the orientation of his physician. Frequently a clergyman may be the one who determines which approach will be selected. Often several have to be attempted before a successful program is arrived at. In any case, recognition of the basic emotional nature of the disability and the inclusion of a program specially directed towards its correction is vital in any successful treatment regimen for the alcoholic. Within the framework of this concept, the use of appropriate drugs to achieve specific objectives is a valid aspect of the total treatment. In certain instances, the successful treatment of an individual may, to a great extent, depends on the use of the right drug at the right time. A critical evaluation of the various drugs recently used in the treatment of alcoholism will be attempted. Those which have, in our experience, proved useful will be described in detail and a realistic appraisal of the objectives of the various drug therapies presented.

Drugs used in the treatment of alcoholism can be broadly classified [3] in accordance with the objectives sought through their use. The accompanying table groups the drugs whose therapeutic objectives are more or less the same (Table 1).

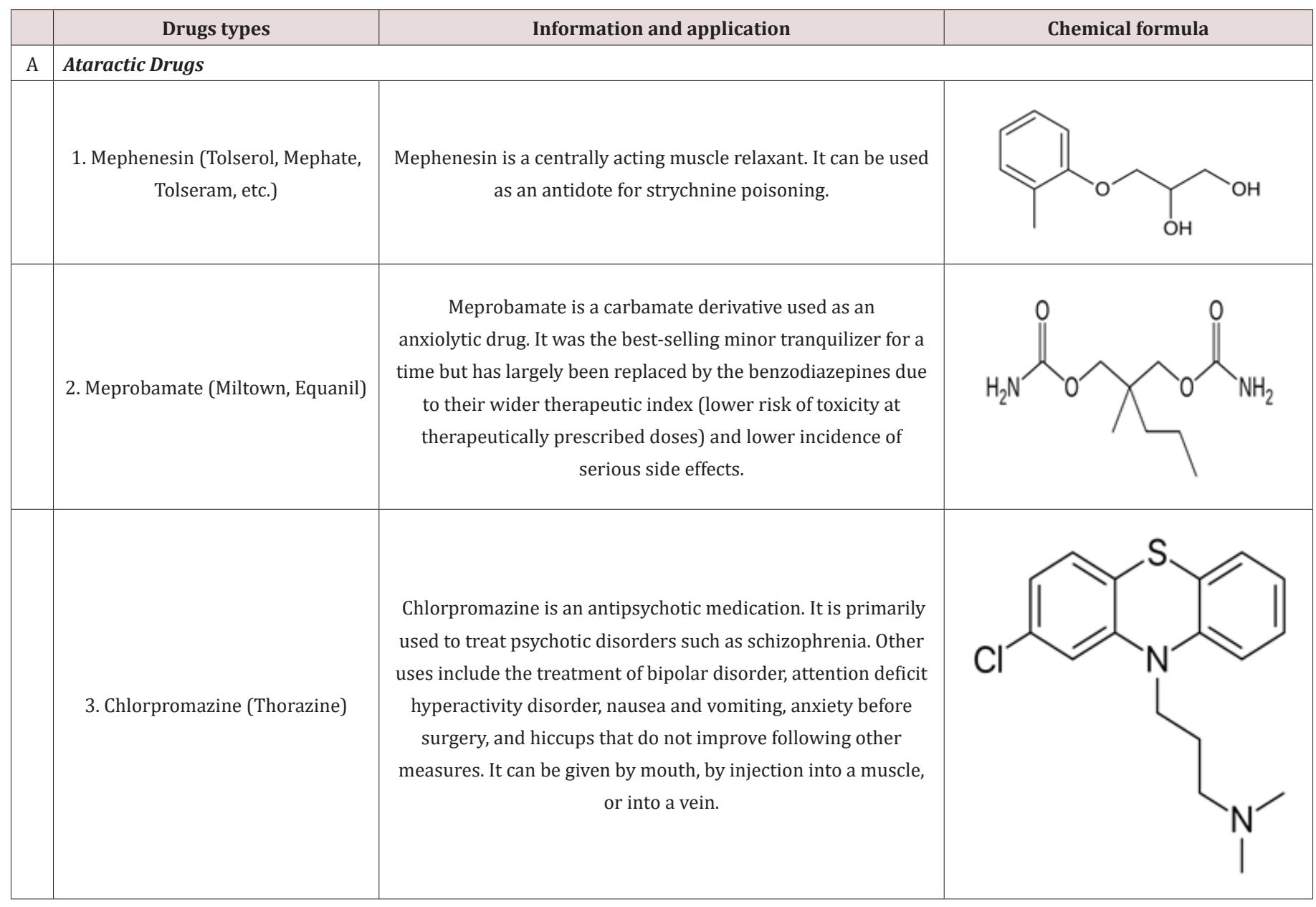




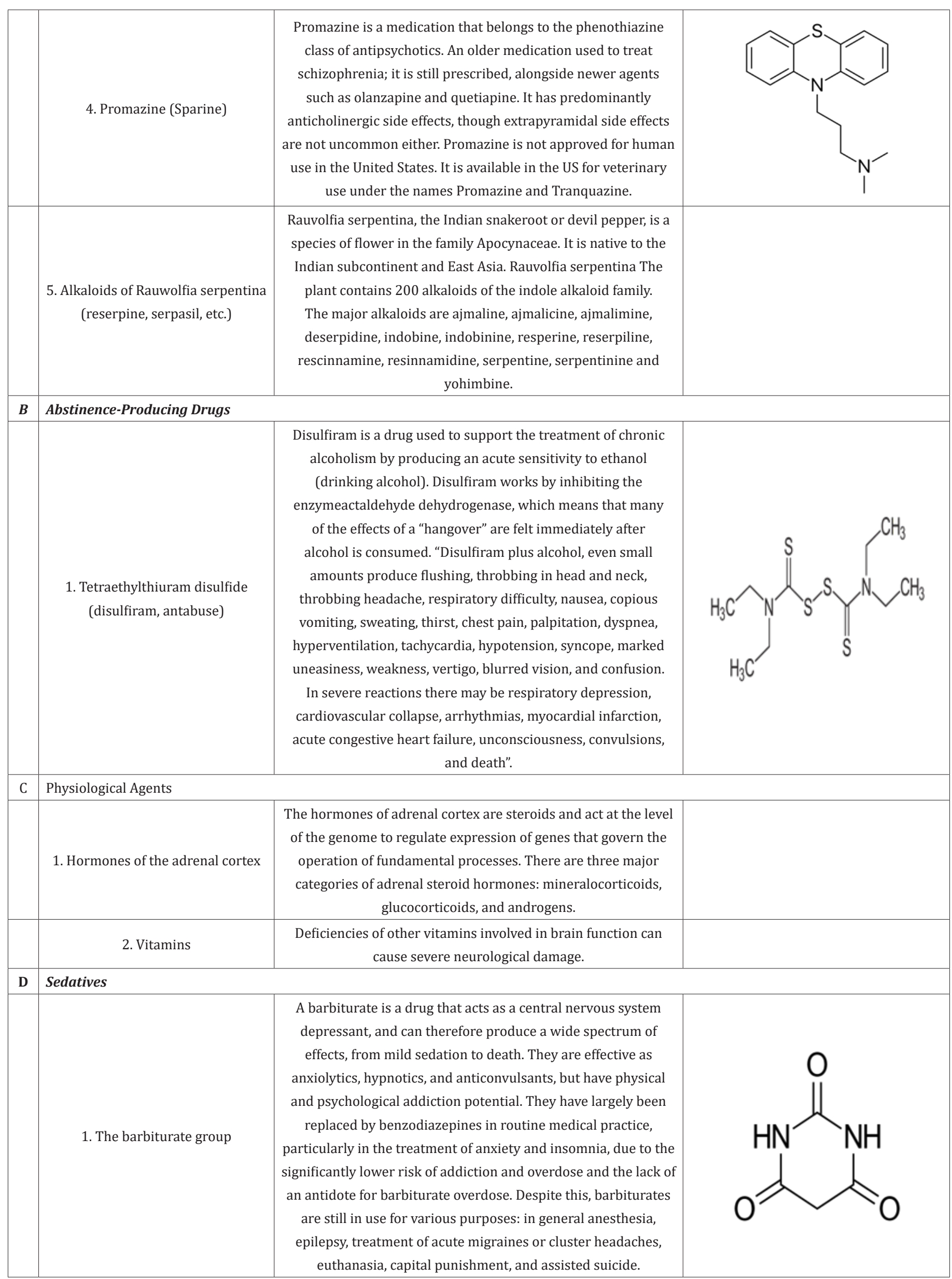




\begin{tabular}{|l|l|}
\hline 2. Paraldehyde & $\begin{array}{c}\text { Paraldehyde is the cyclic trimer of acetaldehyde molecules. } \\
\text { Formally, it is a derivative of 1,3,5-trioxane. The corresponding } \\
\text { tetramer is metaldehyde. A colorless liquid, it is sparingly } \\
\text { soluble in water and highly soluble in ethanol. Paraldehyde } \\
\text { slowly oxidizes in air, turning brown and producing an odor of } \\
\text { acetic acid. It quickly reacts with most plastics and rubber. It is a } \\
\text { central nervous system depressant and was soon found to be an } \\
\text { effective anticonvulsant, hypnotic and sedative. It was included } \\
\text { in some cough medicines as an expectorant (though there is no } \\
\text { known mechanism for this function beyond the placebo effect). }\end{array}$ \\
\hline
\end{tabular}

\section{Drugs Classification}

\section{Ataractic Drugs}

In recent years a significant number of drugs have appeared whose purpose is to decrease or abolish those physical and mental symptoms, such as anxiety, hyperirritability, tremors and restlessness, generally grouped together as tension states. When one considers the frequency with which these symptoms are encountered in the alcoholic, and the difficult problems posed by them in his management, any medication that might reduce or abolish such symptoms with a reasonable degree of safety would be an important factor in the total therapeutic approach. The use of tranquilizing drugs has significantly influenced the therapy of alcoholism and made possible results which in many instances would not be expected. The ataractic drugs are useful in the control of anxiety and hyperirritability at any point in the course of this disease, but they have proved of particular value in the management of the acute alcoholic episode and in the difficult problems that may be present in the immediate post withdrawal period.

\section{Mephenesin}

This substance is essentially a muscle relaxant which functions through an inhibitory effect on the internuncial system of the brain and spinal cord [4]. It also possesses a tranquilizing property, probably through a central effect. Mephenesin has been combined with carbamate for longer effective blood levels (e.g., Tolseram) and with glutamic acid hydrochloride (Mephate) for more efficient absorption from the gastrointestinal tract. Its use in the treatment of alcoholism is based on its inhibition of gross tremor, frequently a disturbing symptom in the acute and post withdrawal phases of alcoholism, and its anxiety-allaying properties. By and large, the results of treatment have been only fair. It has had some value as an adjunctive treatment of those phases noted above, but its effect has been far from uniform. Certain individuals have described considerable benefit from mephenesin, particularly in regard to the diminution or disappearance of disturbing tremor; in other instances, no subjective or objective improvement has been noted. It has not been particularly helpful in the control of anxiety during abstinence, a condition that frequently forebodes the resumption of drinking. The drug and its combinations appear to be quite safe. It is best administered orally after meals or with milk or fruit juice to avoid possible gastrointestinal disturbances. The pure drug may be given intravenously. Initial dosage during acute symptoms should be fairly large. One to 3 grams of mephenesin or Tolseram, and 0.5 gram of Mephate, given several times during the day, is usually adequate. The dose should be decreased as the patient's symptoms decrease. The size of the maintenance dose must be individually determined. The value of long-term treatment with mephenesin for the control of anxiety and tension is not clear-cut $[5,6]$, but occasionally patients have stated that they have found such treatment quite helpful.

\section{Meprobamate}

This substance is a synthetic compound related structurally to mephenesin but having far more potent tranquilizing effects. Its exact physiological and pharmacological actions are not yet clearly understood [6], but there is not much doubt that it has an effect on the higher centers of the nervous system, and it has been recently shown to reduce the frequency and amplitude of the action potential of striated muscle as measured by the electromyogram [7]. Meprobamate is commercially available as Miltown or Equanil. Meprobamate has proved a valuable adjunct in the treatment of chronic alcoholism through its effect in controlling the post withdrawal irritability and in allaying the recurrent anxiety and tension frequent during the early abstinence period. In a significant percentage of cases of moderate severity, abrupt withdrawal of alcohol on an ambulatory basis can be accomplished through its use [8]. Disturbing symptoms under such circumstances may be reduced sufficiently to fall within the patient's ability to tolerate them without drinking.

The use of meprobamate in the ambulatory treatment of the drinking phase implies a cooperative patient who wants sincerely to stop the drinking bout. Its use is not feasible in the unmotivated patient, or the individual who is too drunk to cooperate in the treatment program. Its value is further enhanced by its soporific action, making the use of sedatives less necessary or unnecessary [6]. It has been in the control of recurrent anxiety during abstinence, that meprobamate has proved most useful. The overwhelming need to escape from intolerable anxiety is the most frequent cause of the resumption of drinking during abstinence. The use of meprobamate is often as effective as the use of alcohol in the reduction of these feelings to the patient's frustration tolerance. The advantage of the continued abstinence is self- 
evident from the practical point of view of job and family as well as from the continuing opportunity afforded for psychotherapy or other approaches to the underlying emotional problems. Since untoward side reactions are insignificant, most patients can be kept on treatment for as long as necessary with safety. Many patients will resume taking meprobamate independently with any return of anxiety as a substitute for alcohol. The drug is supplied in $400 \mathrm{mg}$. tablets. For abrupt withdrawal 1600 to $2400 \mathrm{mg}$. daily in divided doses appears to be adequate and safe. The amount necessary for maintenance or for intermittent use during periods of abstinence must be individually determined. As little as $400 \mathrm{mg}$. per day may be effective.

\section{Chlorpromazine}

Chlorpromazine (Thorazine) has been used in innumerable conditions, both of physical and psychological origin, in the past few years. The literature [9] contains ample data concerning its pharmacological and physiological characteristics as well as its side effects and complications. All of these data are applicable to the use of chlorpromazine in the treatment of alcoholism. Chlorpromazine has been of the greatest value in the treatment of the acute drinking phase and in the control of disturbing symptoms of the post withdrawal syndrome. Indeed, it has sufficiently enhanced treatment in these areas so that the abrupt withdrawal of alcohol from the acute alcoholic (in our opinion the treatment of choice) can be carried out in the environment of the patient's home in a great many instances [10]. The profound tranquilizing effect of chlorpromazine can so depress the psychomotor hyperactivity that the patient is scarcely aware of its existence. The antiemetic properties of the drug give it further value in a condition where nausea, vomiting and retching may be prominent symptoms.

At the onset of treatment of chlorpromazine should be given in sufficiently large doses to insure adequate depression of activity and sleep if possible [9]. The oral or parenteral route may be used depending on the patient's condition. When a patient is in coma or stupor when first seen, chlorpromazine of course should be withheld until sufficient activity develops to warrant its use. The size of the initial dose will depend on the condition of the individual patient, the degree of psychomotor hyperactivity being the most important consideration. Doses of $75 \mathrm{mg}$. to $100 \mathrm{mg}$. are usually adequate although on occasion more may be necessary [11]. Following the initial dose, subsequent therapy will depend on the needs of the individual being treated. Such factors as subjective feelings of anxiety, tremor and jitteriness will influence the dosage. Patients should be kept on treatment for four to seven days. The amount of the drug is reduced as the patient improves, and usually $25 \mathrm{mg}$., three or four times daily is sufficient to maintain a state of tranquility. On such a regimen the patient is usually cooperative and relaxed. Fluids and other necessary substances can be administered orally and the need for barbiturate sedation, always a danger in the alcoholic, is drastically reduced or eliminated.
It should be mentioned that because of the hypotensive effect of chlorpromazine some patients may develop severe postural hypotension particularly during the first few days of therapy. Those concerned with taking care of the patient should be warned of the possibility. The presence of liver disease in a sizable percentage of alcoholics does not seem to be a contraindication to the use of chlorpromazine for the short period of time necessary in alcoholism [11]. The use of long-term maintenance doses of chlorpromazine does not seem to be particularly advantageous. Anxiety and tension during the abstinence period can usually be adequately controlled by other tranquilizers with fewer unwanted side effects. It is well to mention here the decreased frequency with which delirium tremens is seen in patients abruptly withdrawn from alcohol with the aid of chlorpromazine.

\section{Promazine}

Promazine (Sparine) has been used in the treatment of alcoholism [12]. The indications for its use are identical with those of chlorpromazine. An initial dose of from 25 to $100 \mathrm{mg}$. seems adequate and the drug is repeated every four to six hours as the condition of the patient warrants. The drug may be given parenterally when the patient is unable to take it orally. If anything, the drug seems more effective than chlorpromazine in controlling psychomotor hyperactivity and other symptoms of the acute and post withdrawal phases [12]. The hypotension and compensatory tachycardia are less pronounced and gastric symptoms are efficiently controlled. The presence of severe liver disease seems to offer no contraindication. Although experience with promazine has not been wide as yet, all observers seem to concur with the impressions stated above.

\section{Rauwolfia Serpentina}

The alkaloids of Rauwolfia (Reserpine, Serpasil, etc.) have been evaluated as therapeutic agents in the management of alcoholism [13]. In general, their tranquilizing properties have not been of sufficient intensity to be effective in the control of the disturbing symptoms associated with the acute phase. In some instances [14], control of mild anxiety appearing during the course of long-range treatment in the abstinent alcoholic has resulted from their use. In general, other tranquilizing agents have been more successful and the Rauwolfia alkaloids have become increasingly less important in this condition [13]. They should, however, be kept in mind for use in those patients in whom the other tranquilizing drugs are poorly tolerated.

\section{Abstinence-Producing Drugs \\ Tetraethylthiuram Disulfide (Disulfiram, Anlabuse)}

The chance discovery in Denmark of the incompatibility of tetraethylthiuram disulfide (TETD) and alcohol in the human organism has led to the development of a drug which, when properly used in properly selected patients, offers a powerful and efficient tool in the treatment of chronic alcoholism [15]. TETD has 
the capacity of inhibiting the metabolism of alcohol in the human body at the acetaldehyde stage. The increasing concentration of this substance as a result of the metabolic block results in the development of unpleasant physical symptoms which makes further drinking virtually impossible. The drug in effect seems to be a "built-in policeman." The advantage of this phenomenon in the treatment of the alcoholic who sincerely wants to stop, but for a variety of reasons cannot maintain abstinence, is obvious. Removing the factor of choice of drinking or not drinking, in itself, frequently results in the alleviation of much tension and anxiety. A period of at least 48 to 96 hours following cessation of treatment is necessary to "clear" the patient so that he can drink without toxic effects. This constitutes a built-in "cooling-off" period, during which a solution, other than drinking, may be found for the problems, real or fancied, disturbing the patient. TETD offers the patient a clear-cut, realistic and practical reason for not drinking.

\section{Methods of Administration}

TETD is available in the United States under the proprietary name "Antabuse" in tablets of 0.5 gram. The drug is administered by mouth. When TETD was first available, its use was frequently associated with unpleasant side effects. Subsequent experience has shown that most of the undesirable symptoms could be eliminated or markedly decreased by a reduction in dosage, which did not impair the therapeutic efficiency of the drug. A single daily dose of 0.5 gram is considered a satisfactory one. This amount of TETD will insure a significant alcohol-TETD reaction in the great majority of individuals. A small percentage of patients may experience mildly unpleasant side reactions on this dosage and in these cases the dose can be reduced to 0.25 gram or even 0.125 gram daily with sufficient "protection." Since there is no cumulative effect, the drug is administered without intermission, and for as long as is necessary in the opinion of the physician. No late complications have been ascribed to TETD, even after two years or longer of continuous treatment. The drug should be taken at a regular time so that it will become a part of the daily habit pattern. The time of the morning ablutions or just before retiring seems to be most practical. In the latter instance the occasional drowsiness that results can be taken advantage of [16].

\section{Side Effects, Toxicity and Contraindications}

Many side effects were described in the early literature [17]. Most of these, as has been previously stated, resulted from overdosage. Most common are drowsiness, headache, gastrointestinal complaints and fatigability. Diminished sexual potency and a transient rash (which seems to be an allergic phenomenon) are occasionally seen. Most of these occur in the first few days of treatment and usually disappear spontaneously. Sometimes a reduction in dosage is necessary. It is most rare to have to discontinue treatment entirely. The only significant toxic effect of TETD alone is psychosis [18]. Its development is a clear contraindication to the use of the drug. Although an incidence of psychosis of up to 10 per cent was described in the early days of high dosage, it is extremely rare on present schedules. A history of a psychotic episode in the past should make one extremely wary of instituting TETD therapy. The psychotic episode usually clears up in a period of one to three weeks after stopping treatment. When TETD was first introduced a number of conditions were considered contraindications of its use. Among them were liver disease [19], thyrotoxicosis [20], diabetes mellitus [21], epilepsy and cardiovascular disease [22], particularly if the coronary arteries were involved. In light of continuing experience, none of these diseases is in itself a contraindication when a real indication for TETD exists. If one is aware of the existence of these diseases at the onset of TETD treatment they can be adequately managed. The importance of the patient's alcoholism and its social and physical impact should be the criteria used in determining whether or not TETD will be used in the face of coexisting disease states.

The TETD-Alcohol Reaction. Clinically the reaction is first manifested by flush, dyspnea, headache and tachycardia [23]. The sclerae become injected ("bull eye") and a sense of substernal discomfort may be present. This reaction occurs usually within 30 minutes of the ingestion of alcohol. The reaction may gradually disappear, and the patient falls into a deep sleep, or it may progress to exhibit the "late" manifestations. These are principally gastrointestinal with nausea and vomiting prominent and those of hypotension, with pallor, cerebral anoxia and ultimately collapse. These latter symptoms can be quite severe and in such an instance the presence of an impaired coronary circulation may lead to complications including myocardial infarction. The antihistamines are a specific antidote and rapidly remove the symptoms of the alcohol-TETD reaction through a specific blocking effect. They at the same time enhance the soporific effects so that the patient falls into a deep sleep. Thus, the reaction can be stopped by the use of antihistamines and, when severe, rapidly through their parenteral use. Quick-acting antihistamines in fairly large doses can be used to abort an already existing TETD alcohol reaction [24]. The value of TETD is not materially lessened by the effect of antihistamines [24]. The combination of alcohol and antihistamine in the TETD-treated patient will usually result in a deep sleep and not an alcoholic episode of uncontrolled proportions.

The question of a planned TETD-alcohol reaction is a moot one. In the early days "test" reactions were carried out fairly commonly, their purpose being to demonstrate the consequences to the patient. Further experience has shown that in the great majority of instances, it is unnecessary. A clear description of the reaction, presented honestly, seems to be as effective as trial reaction. This is borne out by the fact that the incidence of drinking while on treatment is roughly the same whether or not a trial reaction was experienced. When a patient with coronary disease is treated with TETD, the increased hazard of an alcohol reaction must be strongly impressed upon him, and a trial reaction is obviously contraindicated under these circumstances. When, because of the 
personality or desire of certain patients, a trial reaction is decided upon it should always be done in a hospital where adequate facilities for treating a severe reaction, if one should occur, are available. It is wise to have the patient on TETD for one or two weeks prior to the test. An ounce of whiskey or the equivalent is usually sufficient to provoke a reaction, but on occasion it may have to be repeated in 30 minutes. Patients on TETD should carry a card on their person so stating and describing the antidote (antihistamine). This helps avoid the possibility of additional alcohol being administered by well-intentioned but uninformed people, in the event of a reaction due to drinking. When properly used, TETD offers a real advantage in the treatment of chronic alcoholism. No serious complications have resulted on current dosage schedules. Arrest and rehabilitation in previously "untreatable" alcoholics have been accomplished through its use.

\section{Physiologic Agents}

\section{Hormones}

The endocrine nature of chronic alcoholism has been considered a possibility for some time. With the increased activity stemming from the newer knowledge of the adrenal cortical steroids, renewed interest in the relationship of the pituitary-adrenal axis and chronic alcoholism was aroused [25]. In the main, two groups of thought developed; on the one hand, chronic alcoholism was felt to be purely a clinical expression of an existing state of hypoadrenalism and, as such, "cure" would result from the correction of this defect; on the other, the benefits described to the use of the cortical steroids were felt to be due to their effect on the stress phenomena resulting from chronic alcoholism. Subsequent studies have failed to disclose any real evidence for a pure endocrine etiology of alcoholism [26], and most workers no longer seriously consider this. However, there is little question that the use of cortical steroids has been of value in the treatment of certain phases of alcoholism, most notably in acute alcoholism, the post withdrawal syndrome and delirium tremens [27]. In these conditions, the cortical steroids have been most effective in controlling symptoms, shortening the duration of disability and, in the case of delirium tremens, possibly life-saving. It is our feeling that this improvement results from the effect of the steroids on the phenomena occurring as a result of the severe stress that alcoholism induces in the organism. Beneficial results [28] have been observed both with adrenocorticotrophic hormone, the cortical steroids themselves and whole cortical extracts. Dosage levels similar to those used in other acute disease states are recommended. The steroids are valuable for the acute phases of alcoholism and no advantage results from continuing their use into the long-range treatment program. With the availability of effective ataractic drugs, the use of adrenal steroids in alcoholism has decreased. Equally good results are obtained with the latter with greater ease of administration and freedom from the possibility of the physiological disturbances which may result from the use of the steroids.

\section{Vitamins}

Vitamins have for so long been a part of the therapeutic armamentarium against alcoholic disease that any discussion of therapy would be incomplete without their being mentioned. The use of vitamins of the $\mathrm{B}$ group has been traditional in the treatment of alcoholism [29]. Such chronic complications as Wernicke's syndrome, peripheral neuropathy and the alcoholic encephalopathy of Jolliffe seem to be markedly benefited by large amounts of B group vitamins [30]. More recently, because of the possible involvement of the adrenal, vitamin C (ascorbic acid) in significant amounts has been added to vitamin therapy in alcoholism [31]. Because of the fact that many alcoholics have been on inadequate diets for variable periods, the use of these vitamins is warranted in the treatment of the acute phases. In addition to the correction of the relative state of avitaminosis, their effect on the liver and the adrenal gland is a beneficial one. The oral or intravenous route may be used depending on the status of the patient. Dosage should be at really significant levels to be of any value. The use of $\mathrm{B}$ vitamins in such conditions as neuropathy (thiamine), Wernicke's syndrome (B complex but particularly thiamine and nicotinic acid) and encephalopathy (nictonic acid) constitutes indicated specific treatment [32]. Mention should be made here of the "nutritional" treatment of alcoholism [33]. This is based on the consideration of alcoholism as a hereditary metabolic disease ("genetotrophic"), resulting from the presence of certain inherited abnormal nutritional needs which are not met by the ordinary diet. Since the specific deficiencies are not known, the use of excessive amounts of known vitamins, minerals and protein should automatically correct them. Alcoholism would then be cured and the alcoholic should then be able to indulge in normal drinking. Most workers have been unable to verify this concept. The use of such a nutritional regimen in itself has had no discernible effect on the course of chronic alcoholism or on the incidence or duration of acute alcoholic relapses.

\section{Sedatives}

The problem of sedation for the alcoholic has always been a thorny one $[34,35]$. The temptation to use sedatives in everincreasing amounts to control the disturbing manifestations of anxiety and tension is a great one. Not infrequently sedatives have been abused in the treatment of alcoholism with resultant addictions and dependencies that are equally or more difficult to manage than the alcoholism. The need for sedation has been greatly diminished through the use of ataractic drugs, but on occasion they are indicated and useful.

\section{Barbiturates}

The barbiturates are the sedatives of choice in the treatment of chronic alcoholism [36]. They should be reserved primarily for insuring a good night's sleep and for use in those cases in which the ataractic drugs alone do not control symptoms sufficiently. The choice of the barbiturate does not seem to make a great difference; 
the preference of the physician or the patient seems to be the deciding factor. The use of the smallest possible effective dose must be stressed. By and large, sedatives are indicated only for persistent insomnia in present regimens. The patient should never be given large amounts of barbiturates to use at his discretion; the subsequent course is most precarious! For the same reason, longterm use of barbiturates is not advisable. It is preferable to issue prescriptions for small quantities to be renewed as indicated and controlled by the physician. An important consideration in the use of barbiturates is to remain constantly aware of the possibility of addiction as a disastrous complication of casual and prolonged use of these drugs.

\section{Paraldehyde}

Paraldehyde has been the most widely used sedative agent for the treatment of acute alcoholism and its immediate sequelae [36]. Despite certain disadvantages, particularly its overpowering odor, it has been an efficient agent. Its use, orally or rectally, has often been the only means of managing an acutely disturbed alcoholic. The efficiency of the ataractic drugs and the cortical steroids has made the use of paraldehyde far less necessary than previously. On occasion it may still be a useful agent. As with the barbiturate sedatives the possibility of habituation or addiction must be kept in mind. Paraldehyde "jags" or dependency is not rare phenomenon.

\section{Delirium Tremens}

The use of the tranquilizers and steroids in the treatment of alcoholism has materially decreased the incidence of this serious condition. When it occurs, it constitutes a serious and potentially fatal complication and it must be so viewed. Experience with adrenal steroids has been most gratifying. They control symptoms rapidly and not infrequently have appeared to be life-saving. The dosages used should be in the maximal range and may be given orally or parenterally. Clinical results seem to be comparable with the various steroid preparations. Since the treatment is short-term, routine precautions suffice. Reports on the use of chlorpromazine in the treatment of delirium tremens are equally enthusiastic [37] Enough should be used to adequately control the symptoms, which is a matter of individual variation. In the management of delirium tremens care must be taken to insure adequate fluid and electrolyte intake, and intravenous administration is usually indicated [38] The use of large doses of B vitamins [29], previously a mainstay in the treatment, is still advisable. In our experience, however, treatment with either chlorpromazine or steroid hormones is more dramatic and effective than treatment with fluids, vitamins and sedatives alone.

\section{Summary}

Drug therapy, when appropriately used, is of much value in the total therapeutic attack on alcoholism. The ataractic drugs and the steroid hormones have greatly increased the efficiency of the treatment of the acute phase and complications of chronic alcoholism. Tetraethyl thiuram disulfide offers a mechanical barrier which, in some cases, offers the only way of controlling drinking and thus bringing to the patient a long-range program of rehabilitation. The newer and more traditional drug therapies of alcoholism are described, and their usefulness and limitations discussed.

\section{References}

1. Mason BJ, Quello S, Shadan F (2017) Gabapentin for the treatment of alcohol use disorder. Expert Opinion on Investigational Drugs 27(1): 113-124.

2. Miller NS (1994) Psychiatric diagnosis in drugs and alcohol addiction. Alcoholism Treatment Quarterly 12(2): 75-92.

3. Hildebrandt T, Epstein EE, Sysko R, Bux DA (2017) Using factor mixture models to evaluate the type A/B classification of alcohol use disorders in a heterogeneous treatment sample. Alcoholism: Clinical and Experimental Research 41(5): 987-997.

4. El Balkhi S, Djezzar S, Poncelet L, Dulaurent S, Morichon J, et al. (2016) Abus et intoxication mortelle à la méphénésine (Abuse and lethal intoxication involving mephenesin). Toxicologie Analytique et Clinique 28(1): 85-89.

5. Numazawa T (2016) Treating depression with the mephenesin analog skeletal muscle relaxant methocarbamol. Open Journal of Depression 5(4): 40-47.

6. Ceschi A, Berger D, Dickenmann M, Bodmer M (2013) Pharmacokinetics of meprobamate in overdose treated with continuous venovenous hemodiafiltration (CVVHDF). Hemodialysis International 17(4): 656659.

7. Neirinck L (2013) Meprobamate-containing med withdrawn from Canadian market. Reactions Weekly 1469(1): 2-2.

8. Felby S (2009) Concentrations of meprobamate in the blood and liver following fatal meprobamate poisoning. Acta Pharmacologica et Toxicologica 28(5): 334-337.

9. Payseur J, Wu J, Bahinski A, Rossman E (2017) Combined cardiovascular, respiratory, and neurobehavioral telemetry model in the conscious rat: a novel approach to study the acute physiological effects of caffeine and Chlorpromazine Following Oral Administration. Journal of Pharmacological and Toxicological Methods 88: 209.

10. Aykut D, Karaguzel E, Karaguzel E (2017) Antipsychotics, chlorpromazine and recurrent priapism: a review over a case. Anatolian Journal of Psychiatry 18(Ek.1): 43-45.

11. Gailis L, Tourigny A (1984) Chlorpromazine and dithioerythritol protection against acute ethanol toxicity. Alcoholism: Clinical and Experimental Research 8(3): 308-313.

12. El-Bassiouny O, El Taher EM, Abd El-Ghaffar MEED (1992) Alfentanil/ promazine versus meperidine/promazine as a sedative regimen during local analgesia for cataract operation. Documenta Ophthalmologica 82(3): 201-210.

13. Vakil R (2009) Rauwolfia Serpentina treatment of high blood pressure. Acta Medica Scandinavica 152(2): 107-114.

14. Rai A, Kumar S, Bauddh K, Singh N, Singh RP (2017) Improvement in growth and alkaloid content of Rauwolfia serpentina on application of organic matrix entrapped biofertilizers (Azotobacter chroococcum, Azospirillum brasilense and Pseudomonas putida). Journal of Plant Nutrition 40(16): 2237-2247.

15. Tampier L, Quintanilla ME, Israel Y (2008) Tolerance to disulfiram induced by chronic alcohol intake in the rat. Alcoholism: Clinical and Experimental Research 32(6): 937-941.

16. Prakash S, Ambekar A, Dayal P (2015) Occasional alcohol use, relapse to opioids and the role of disulfiram. Alcoholism: Journal of Substance Use 21(3): 228-229. 
17. Christensen JK, Rønsted P, Vaag UH (1984) Side effects after disulfiram. Acta Psychiatrica Scandinavica 69(4): 265-273.

18. Jadhav PB (2016) Disulfiram-induced toxic pustuloderma (Acute Generalized Exanthematous Pustulosis). Indian Journal of Drugs in Dermatology 2(1): 31 .

19. Iber FL, Chowdhury B (2008) The persistence of the alcohol-disulfiram reaction after discontinuation of drug in patients with and without liver disease. Alcoholism: Clinical and Experimental Research 1(4): 365-370

20. Bawa F, Kalathil D, McNulty S (2016) A different cause of thyrotoxicosis: Alemtuzumab induced thyrotoxicosis. Endocrine Abstracts 44: EP93.

21. Aljerf L, Alhaffar I (2017) Salivary distinctiveness and modifications in males with Diabetes and Behçet's disease. Biochemistry Research International 2017: 1-12.

22. Krag B, Dam M, Angelo H, Christensen JM (1981) Influence of disulfiram on the serum concentration of carbamazepine in patients with epilepsy. Acta Neurologica Scandinavica 63(6): 395-398.

23. Bourcier S, Mongardon N, Daviaud F, Moachon L, Arnould MA, et al (2013) Disulfiram ethanol reaction mimicking anaphylactic, cardiogenic, and septic shock. American Journal of Emergency Medicine 31(1): 270. e1-270.e3.

24. Martensen-Larsen $O$ (1956) The treatment of acute alcohol intoxication with antihistamine, disulfiram, fructose (honey) and vitamin B6. Acta Psychiatrica Scandinavica 31(S106): 343-345.

25. Mezey E (2000) Influence of sex hormones on alcohol metabolism. Alcoholism: Clinical and Experimental Research 24(4): 421.

26. Erol A, Ho AMC, Winham SJ, Karpyak VM (2017) Sex hormones in alcohol consumption: a systematic review of evidence. Addiction Biology p. 1-13.

27. Moore DT, Fuehrlein BS, Rosenheck RA (2017) Delirium tremens and alcohol withdrawal nationally in the Veterans Health Administration. The American Journal on Addictions 26(7): 722-730.
28. Erwin D (2007) Adrenocorticotrophic hormone in the treatment of spinal headache. Anaesthesia 40(2): 200.

29. Beulens JWJ, Schaafsma G, Kok FJ, Hendriks HFJ (2004) Kinetics of plasma homocysteine and status of B-vitamins in moderate alcohol consumers. Alcoholism: Clinical \& Experimental Research 28: 7A.

30. Cravo ML, Camilo ME (2000) Hyperhomocysteinemia in chronic alcoholism: relations to folic acid and vitamins b6 and b12 status. Nutrition 16(4): 296-302.

31. Hoyumpa AM (1986) Mechanisms of vitamin deficiencies in alcoholism. Alcoholism: Clinical and Experimental Research 10(6): 573-581.

32. Kühn AL, Hertel F, Boulanger T, Diederich NJ (2012) Vitamin B1 in the treatment of Wernicke's encephalopathy due to hyperemesis after gastroplasty. Journal of Clinical Neuroscience 19(9): 1303-1305.

33. Faetz J (1998) Nutritional therapy: the missing link to treatment of alcoholism. Addictions Nursing 10(4): 197-200.

34. Wise J (2015) Routine use of sedatives before surgery has little benefit, study shows. BMJ 350: h1158-h1158.

35. Safar P, Bleyaert A, Detre K, Moossy J, Reinmuth O (1986) Cerebral resuscitation with Barbiturates. Anesthesiology 65(20): 235

36. Philips GW, Carmichel EB, Kay FA (1944) Studies on paraldehyde. II. The median lethal dose, LD of paraldehyde for both young and old rats on two dietary regimes. Anesthesiology 5(3): 287-290.

37. Kalda R (1973) Chlorpromazine contraindicated in Delirium Tremens. American Journal of Psychiatry 130(9): 1042-1042.

38. Wojnar M, Bizon Z, Wasilewski D (1999) Assessment of the role of kindling in the pathogenesis of alcohol withdrawal seizures and Delirium Tremens. Alcoholism: Clinical \& Experimental Research 23(2): 204-208.

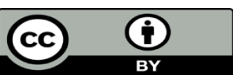

This work is licensed under Creative Commons Attribution 4.0 License

Submission Link:

Submit Article

DOI: 10.32474/DDIPIJ.2018.02.000130

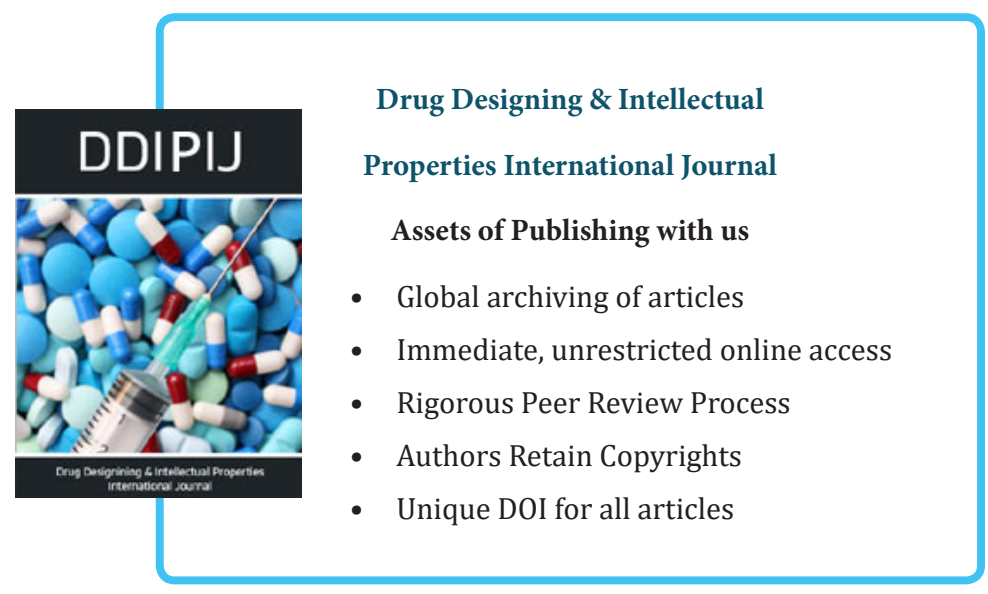

